\title{
Índices hematimétricos associado as parasitoses intestinais no Município de Breves, Ilha do Marajó, Pará
}

\author{
Hematimetric indexes associated with intestinal parasites in the municipality of Breves, Ilha do \\ Marajó, Pará
}

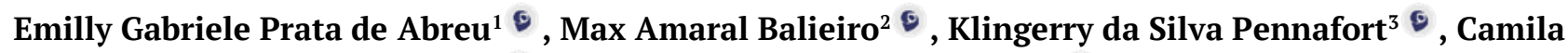 Rodrigues Barbosa Nemer ${ }^{3} \bullet$, Edmundo de Souza Moura Filho ${ }^{3}$, , Rubens Alex de Oliveira Menezes $^{3^{*}} \bullet$

\footnotetext{
${ }^{1}$ Acadêmica de Enfermagem e Bolsista de Iniciação Cientifica PROBIC da Universidade Federal do Amapá, Macapá, Amapá. Brasil.

${ }^{2}$ Acadêmico de Enfermagem da Universidade Federal do Amapá, Macapá, Amapá. Brasil. ${ }^{3}$ Departamento de Ciências Biológicas e da Saúde da Universidade Federal do Amapá, Docente do curso de Enfermagem e Orientador de Iniciação Cientifica PROBIC da Universidade Federal do Amapá, Macapá, Amapá, Brasil. *Autor para correspondência. E-mail: ramenezes@hotmail.com/rubens.alex@unifap.br
}

\begin{abstract}
Resumo: Introdução: As parasitoses intestinais, principalmente em países subdesenvolvidos, constituemse uma grande problemática de saúde, sendo estas causadas por protozoários e helmintos decorrentes das inadequadas condições sócio sanitárias em que a população está inserida. Objetivo: Identificar a incidência de enteroparasitas e sua relação com anemia de uma população do Bairro Jardim Tropical, Breves - PA. Material e Métodos: Estudo transversal realizado através de exames de fezes pelos métodos direto e de Hoffman, questionário estruturado, e coleta sanguínea para análise hematológica, sendo coletados de 83 amostras de residentes da localidade. Resultados: O estudo evidenciou 30,1\% (25/83) das amostras coletadas com índices hematimétricos sugestivos de anemia. Os resultados apresentaram positividade de $75,6 \%$ (63/83), sendo, a maioria dos infectados do sexo feminino $65 \%$ (41/63), contra $34,9 \%$ (22/63) do sexo masculino. Em relação à intensidade das infecções, o poliparasitismo 73\% (46/63) prevaleceu frente ao monoparasitismo 26,9\% (17/63). Adicionalmente, foi observado uma maior incidência de protozoários em relação aos helmintos, tendo como agentes etiológicos os protozoários patogênicos: Entamoeba histolytica/ E. díspar 46\% (29/63) e a Giardia intestinalis 7,9\% (5/63). Para helmintos a maior incidencia foi de Trichuris Trichiura 31,7\% (20/63), seguido de Ascaris lumbricoides 20,6\% (13/63). Ademais, foram encontrados uma elevada incidência de protozoários não patogênicos como: Entamoeba coli, Endolimax nana, Iodamoeba butschlii e Blastocistis hominis. Considerações finais: Os resultados reforçam a necessidade da implementação de políticas públicas eficazes, visando garantir saneamento básico de qualidade à população.
\end{abstract}

Palavras-chave: anemia, Amazônia, enteroparasitas, ribeirinho, saneamento básico.

\begin{abstract}
Introduction: Intestinal parasites, especially in underdeveloped countries, constitute a major health problem, which are caused by protozoa and helminths resulting from inadequate socio-sanitary conditions in which the population is inserted. Objective: To identify the incidence of intestinal parasites and their relationship with anemia in a population of Bairro Jardim Tropical, Breves - PA. Material and Methods: Cross-sectional study performed through stool examinations by direct and Hoffman methods, structured questionnaire, and blood collection for hematological analysis, being collected from 83 samples of local residents. Results: The study showed 30.1\% (25/83) of the samples collected with blood counts suggestive of anemia. The results showed a positivity of $75.6 \%$ (63/83), with the majority of infected females being $65 \%$ (41/63), against 34.9\% (22/63) of males. Regarding the intensity of infections, polyparasitism $73 \%$ (46/63) prevailed compared to monoparasitism $26.9 \%$ (17/63). Additionally, a higher incidence of protozoa than helminths was observed, with pathogenic protozoa as etiological agents: Entamoeba histolytica/ E. dispar 46\% (29/63) and Giardia intestinalis 7.9\% (5/63). For helminths, the highest incidence was Trichuris Trichiura 31.7\% (20/63), followed by Ascaris lumbricoides 20.6\% (13/63). Furthermore, a high incidence of non-pathogenic protozoa was found, such as: Entamoeba coli, Endolimax nana, Iodamoeba butschlii and Blastocistis hominis. Final considerations: The results reinforce the need to implement effective public policies, aimed at guaranteeing quality basic sanitation to the population.
\end{abstract}

Keywords: anemia, Amazon, enteroparasites, ribeirinho, basic sanitation. 


\section{Introdução}

A carência principalmente de ferro é apontada como causa prevalecente dos quadros anêmicos e é atualmente, a carestia nutricional em primazia no mundo, acometendo países desenvolvidos e em desenvolvimento. Em 2002, a anemia ferropiva foi reputada como um dos principais fatores para o aumento de doenças. (Castro et al., 2011). A etiologia das anemias se caracteriza pela produção anormal de hemoglobina. O ferro é fundamental para síntese de hemoglobina nos eritroblastos, dessa forma, na ausência do ferro a produção de hemoglobina e hemácias torna-se deficiente (Teles, 2018).

Principalmente em países tropicais como o Brasil, as infecções parasitárias têm um papel importante na ocorrência de anemia. As sintomatologias provocadas por infecções de enteroparasitoses incluem diarreia, anemia, colites e desnutrição, má absorção e obstrução intestinal. Helmintos como o Ascaris lumbricoides, Ancilostomídeos, Trichuris trichuria e protozoários como Giardia intestinalis, denominados causadores de anemia, uma vez que alteram o perfil nutricional do hospedeiro afetando a absorção dos nutrientes (Sucasas et al., 2017).

Populações com difícil acesso ao saneamento básico e à educação tornam-se mais propensos às infecções, posto que a transmissão desses agentes apresenta-se conexa às condições sociais. As doenças parasitárias caracterizam um fator indistinto e alarmante na realidade brasileira, uma vez que o país ainda apresenta déficit de infraestrutura sanitária, sendo responsável pela veiculação destes patógenos através da veiculação hídrica e alimentar (Sucasas et al., 2017).

Embora apresentem ampla distribuição geográfica, a presença, duração e intensidade dessas infecções são influenciadas por características ambientais, domiciliares, nutricionais e outras vinculadas à pobreza. (Ignacio et al., 2017). A comum transmissão orofecal desta patologia corrobora com a necessidade de ampliações de serviços de saneamento básico e de programas de educação sanitária e ambiental (Maia et al., 2015).

Adicionalmente, paralelo ao progresso científico e tecnológico que possibilitaram importantes avanços na saúde pública, com reduções pouco incisivas na prevalência das enteroparasitoses. Ademais, essas patologias ainda significam um grande desafio para a saúde pública, fazendo com que atualmente, os estudos acerca de parasitoses sejam imprescindíveis (Almeida et al., 2014).

Nesse contexto, conforme dados do Departamento de Informática do Sistema Único de Saúde (DATASUS), em 2014 as parasitoses foram responsáveis por 776.358 internações, representando 7,28\% da morbidade hospitalar, sendo a sexta causa de adoecimento no país (Fraga, 2019). Diante da necessidade de um conhecimento sobre a temática abordada, o que poderá servir de subsídios para novos estudos e fomentar sua discussão, esta pesquisa tem como objetivo identificar a incidência de enteroparasitas e sua relação com anemia em uma população do Bairro Jardim Tropical, Breves - PA.

\section{Materiais e métodos}

\section{Tipo de estudo}

Estudo epidemiológico delineado como descritivo transversal realizado com 83 residentes do bairro Jardim Tropical, município de Breves, no estado do Pará, em janeiro de 2019.

\section{Local da pesquisa}

Breves, município brasileiro do estado do Pará, com área de $9.566,572 \mathrm{~km}^{2}$ e de população de 103.497 habitantes. O município possui flora da Amazônia, com predominância de floresta tropical (Ibge, 2021). Cortado por um braço do rio Parauaú, em sua grande parcela, o bairro Jardim Tropical situa-se em área de ressaca e possui vias de acesso não asfaltadas e sistema de escoamento ausente, aumentando o volume de água parada ao redor das residências.

\section{Critérios de inclusão}

Foram considerados para este trabalho os seguintes fatores como critérios de inclusão: a) residente da área de estudo, b) preenchimento do termo de consentimento livre e esclarecido, c) preenchimento do formulário das informações epidemiológicas, d) consentimento na coleta de material biológico (sangue e fezes).

\section{Critérios de exclusão}

Como critérios de exclusão, foram excluídos da pesquisa residentes menores de 18 anos e que não concordaram com a assinatura do termo de consentimento livre e esclarecido, bem como, do preenchimento das informações epidemiológicas pertinentes ao estudo. 


\section{Coleta de dados e período de estudo}

A coleta transcorreu na residência dos participantes, onde foi justaposto ao formulário epidemiológico. As amostras sanguíneas e fecais foram coletadas e devidamente acondicionadas em coletores estéreis e específicos para cada coleta. O período da coleta de dados ocorreu entre os meses de janeiro e fevereiro de 2019.

\section{Instrumento de coleta de dados}

Os formulários apresentam questões para identificação individual e de hábitos de higiene e rotina de cada participante, além de informações sobre a moradia, tipo de moradia, origem da água, presença ou ausência de filtro de água para consumo, destino dos dejetos sanitários, hábitos alimentares, presença ou ausência de fossas (rudimentares e sépticas), entre outras informações.

\section{Coleta e exame de sangue}

As amostras de sangue foram coletadas por punção venosa a vácuo, sendo coletados oito mililitros de sangue venoso de cada paciente. Quatro mililitros foram distribuídos em um tubo contendo EDTA (ácido etilenodiamina tetracético) para identificar os níveis de hemoglobina e realizar análises hematológicas. Nessa pesquisa foi utilizado o equipamento automatizado (Mindray-BC-3000plus) para a realização do hemograma. O hemograma compreende a contagem das células do sangue periférico (hemácias, leucócitos e plaquetas) e a contagem diferencial dos tipos leucocitários, além da quantidade dos valores da hemoglobina e do hematócrito e ainda o cálculo dos índices hematimétricos (Naoum et al., 2011). A anemia foi avaliada de acordo com os padrões de diagnóstico fornecido pelo equipamento automatizado supracitado que no geral é recomendado pela Organização Mundial da Saúde através de índices de hematócrito e hemoglobina com pequenas variações de acordo com a idade e sexo.

\section{Diagnóstico fecal}

As amostras fecais foram preparadas usando a técnica e/ou métodos a fresco, direto e de Hoffman-Pons e Janer. Para cada amostra, duas lâminas foram examinadas para detecção de parasitas, utilizando microscopia óptica (Nikon, Japão) com ampliações de 100X e 400X. As análises foram realizadas no Laboratório de Análises Clínicas (LAC) e no Laboratório de Estudos Morfofuncionais e Parasitários (LEMP) da Universidade Federal do Amapá.

\section{Aspectos éticos}

A pesquisa seguiu a Resolução $n^{\circ} 466$ de 2012 da Comissão Nacional de Ética em Pesquisa na qual sistematiza as normas éticas de pesquisas envolvendo seres humanos, sendo as atividades desenvolvidas fundamentadas pela Resolução do Conselho Nacional de Saúde (Brasil, 2012).

\section{Resultados}

Dos 83 participantes, 75,9\% (63/83) apresentaram resultados coprológicos positivos para um ou mais parasitas, seguindo-se 73\% (46/63) poliparasitados e 26,9\% (17/63) monoparasitados (Figura 1).

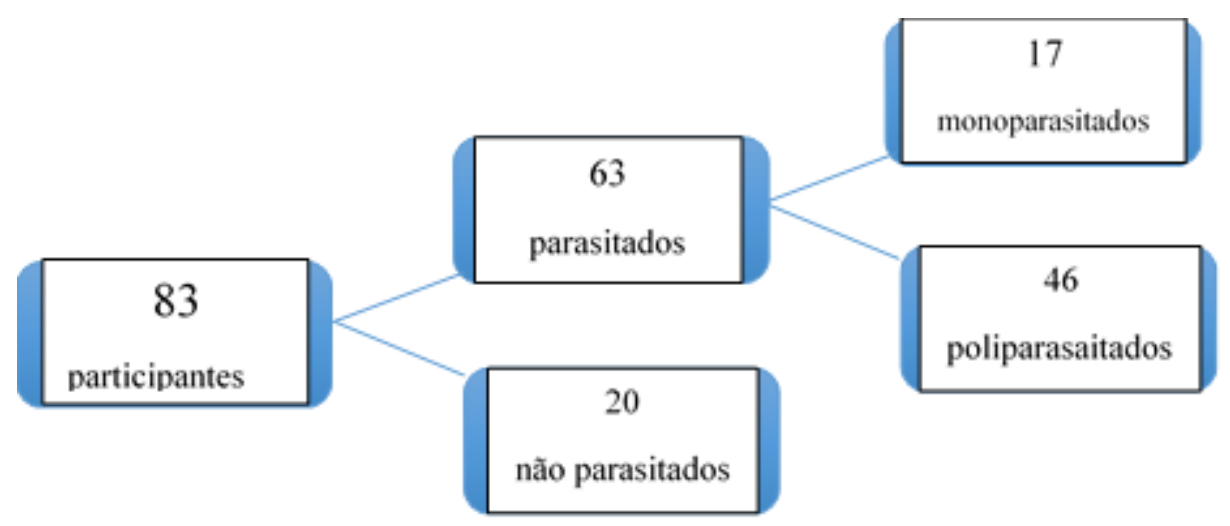

Figura 1. Fluxo de coleta de dados. 
No que tange a tipificação dos agentes etiológicos, identificou-se uma maior incidência de protozoários em relação aos helmintos. Dentre os helmintos, o Trichuris trichiura foi o de maior prevalência com 31,7\% (20/63), seguido do Ascaris lumbricoides 20,6\% (13/63), Ancilostomídeo 1,5\% (1/63) e Hymenolepis nana 1,5\% (1/63). Dentre os protozoários, a Entamoeba histolytica/E. díspar foi a mais frequente 46\% (29/63), seguida pela Endolimax nana 41,2\% (26/63) e a Entamoeba coli 39,6\% (25/63) (Tabela 1).

Tabela 1. Espécimes observados.

\begin{tabular}{|c|c|c|}
\hline $\begin{array}{c}\text { Enteroparasitas } \\
\text { Protozoários }\end{array}$ & Parasitados (n) & Frequência (\%) \\
\hline Entamoeba histolytica/E. díspar & 29 & $46 \%$ \\
\hline Endolimax nana & 26 & $41,2 \%$ \\
\hline Entamoeba coli & 25 & $39,6 \%$ \\
\hline Iodamoeba butschlii & 23 & $36,5 \%$ \\
\hline Blastocistis hominis & 7 & $11,1 \%$ \\
\hline Giardia intestinalis & 5 & $7,9 \%$ \\
\hline \multicolumn{3}{|l|}{ Helmintos } \\
\hline Trichiuris trichiura & 20 & $31,7 \%$ \\
\hline Ascaris lumbricoides & 13 & $20,6 \%$ \\
\hline Ancylostoma duodenale & 1 & $1,5 \%$ \\
\hline Hymenolepis nana & 1 & $1,5 \%$ \\
\hline \multicolumn{3}{|l|}{ Monoparasitismo } \\
\hline Endolimax nana & 5 & $7,9 \%$ \\
\hline Trichiuris trichiura & 4 & $6,3 \%$ \\
\hline Entamoeba histolytica/E. díspar & 4 & $6,3 \%$ \\
\hline Ascaris lumbricoides & 2 & $3,1 \%$ \\
\hline Iodamoeba butschlii & 2 & $3,1 \%$ \\
\hline \multicolumn{3}{|l|}{$\begin{array}{l}\text { Poliparasitismo } \\
\end{array}$} \\
\hline E. coli + Iodamoeba butschlii & 3 & $4,7 \%$ \\
\hline E. coli + E. nana & 2 & $3,1 \%$ \\
\hline E. coli + E. nana $+T$. trichiura $+I$. butschlii + E. histolytica & 2 & $3,1 \%$ \\
\hline E. coli $+A$. lumbricoides $+T$. trichiura $+E$. histolytica & 2 & $3,1 \%$ \\
\hline E. coli + E. nana + T. trichiura $+I$. butschlii $+G$. intestinalis & 1 & $1,5 \%$ \\
\hline A. lumbricoides $+A$. duodenale & 1 & $1,5 \%$ \\
\hline E. nana + A. lumbricoides $+E$. histolytica $+H$. nana & 1 & $1,5 \%$ \\
\hline
\end{tabular}

No que compete a condições socioeconômicas e higiênico-sanitárias, 81,9\% (68/83) inteiraram dispor de núcleo familiar com 4 ou mais pessoas. Relativamente ao nível de escolaridade, grande parte dos partícipes informou não possuir o ensino fundamental completo, tendo este dado uma prevalência de $26,5 \%$ (22/83). Os indivíduos que possuem renda mensal de 1 salário mínimo ou menos ficaram em 67,4\% (56/83). Os que consomem água diretamente do rio constituem-se em 56,6\% (47/83).78,3\% (65/83) habitam em casas de madeira e 56,6\% (47/83) vivem em área de ressaca. Em relação ao destino do lixo, 100\% (83/83) possuem serviço de coleta de resíduos através da prefeitura e 53,0\% (44/83) afirmam usar fossa séptica (Tabela 2).

Supletivamente, das 83 amostras analisadas, 30,1\% (25/83) denotaram índices hematimétricos sugestivos à anemia. $\mathrm{Na}$ análise dos demais índices hematimétricos, examinou-se que $8 \%(2 / 25)$ apresentaram hemoglobina corpuscular média (HCM) abaixo do valor de referência enquanto 56\% (14/25) apresentaram volume corpuscular médio (VCM) acima do valor de referência, sendo 26,0 pg como valor limítrofe inferior e 100,0 fL o valor limítrofe superior, respectivamente (Gráfico 1).

\section{Discussão}

Para quantificar a incidência de casos anêmicos, a concentração de hemoglobina corpuscular média e o valor do hematócrito equivalem aos indicadores hetamológicos do presente estudo. Resultante da redução na concentração de hemoglobina em consequência da carência de ferro, a anemia é tida como um problema de saúde pública, acometendo principalmente adolescentes, crianças, gestantes e lactantes. As parasitoses intestinais são vistas como a principal condição que predispõe a ausência de ferro orgânico. Nada obstante, novos estudos sugerem que quanto a etiologia da anemia, as parasitoses exercem uma relevância secundária (Santos et al., 2016). 
Tabela 2. Condições socioeconômicas e higiênico-sanitárias.

\begin{tabular}{|c|c|c|c|c|}
\hline Variável & *P (n) & "F (\%) & *NP (n) & *F (\%) \\
\hline \multicolumn{5}{|l|}{ Sexo } \\
\hline Masculino & 22 & $26,5 \%$ & 9 & $10,8 \%$ \\
\hline Feminino & 41 & $49,3 \%$ & 11 & $13,2 \%$ \\
\hline \multicolumn{5}{|l|}{ Faixa etária } \\
\hline $18-29$ anos & 19 & $22,8 \%$ & 12 & $14,4 \%$ \\
\hline 30-39 anos & 6 & $7,2 \%$ & 2 & $2,4 \%$ \\
\hline 40-49 anos & 16 & $19,2 \%$ & 3 & $3,6 \%$ \\
\hline $50-59$ anos & 12 & $14,4 \%$ & 1 & $1,2 \%$ \\
\hline Acima de 60 anos & 10 & $12 \%$ & 2 & $2,4 \%$ \\
\hline \multicolumn{5}{|l|}{ Escolaridade } \\
\hline Nunca estudou & 10 & $12 \%$ & 1 & $1,2 \%$ \\
\hline Ensino fundamental incompleto & 17 & $20,4 \%$ & 5 & $6 \%$ \\
\hline Ensino fundamental completo & 7 & $8,4 \% \%$ & 1 & $1,2 \%$ \\
\hline Ensino médio incompleto & 3 & $3,6 \%$ & 1 & $1,2 \%$ \\
\hline Ensino médio completo & 11 & $13,2 \%$ & 6 & $7,2 \%$ \\
\hline Ensino superior & 15 & $18 \%$ & 6 & $7,2 \%$ \\
\hline \multicolumn{5}{|l|}{ Renda mensal } \\
\hline$\leqslant 1$ salário mínimo & 45 & $54,2 \%$ & 11 & $13,2 \%$ \\
\hline > 1 salário mínimo & 18 & $21,6 \%$ & 9 & $10,8 \%$ \\
\hline \multicolumn{5}{|c|}{ Abastecimento de água } \\
\hline Água encanada & 22 & $26,5 \%$ & 13 & $15,6 \%$ \\
\hline Poço artesiano & 1 & $1,2 \%$ & 0 & $0 \%$ \\
\hline Diretamente do rio & 40 & $48,1 \%$ & 7 & $8,4 \%$ \\
\hline \multicolumn{5}{|l|}{ Destinação do lixo } \\
\hline Coleta pública & 63 & $75,9 \%$ & 20 & $24 \%$ \\
\hline \multicolumn{5}{|c|}{ Tipo de domicílio } \\
\hline Madeira & 47 & $56,6 \%$ & 18 & $21,6 \%$ \\
\hline Alvenaria & 2 & $2,4 \%$ & 1 & $1,2 \%$ \\
\hline Mista & 14 & $16,8 \%$ & 1 & $1,2 \%$ \\
\hline \multicolumn{5}{|l|}{ Núcleo familiar } \\
\hline$<4$ & 10 & $12 \%$ & 5 & $6 \%$ \\
\hline$\geqslant 4$ & 53 & $63,8 \%$ & 15 & $18 \%$ \\
\hline \multicolumn{5}{|c|}{ Tipo de terreno } \\
\hline Área de ressaca & 35 & $42,1 \%$ & 12 & $14,4 \%$ \\
\hline Terra firme & 28 & $33,7 \%$ & 8 & $9,6 \%$ \\
\hline \multicolumn{5}{|c|}{ Destino esgotamento sanitário } \\
\hline Fossa séptica & 31 & $37,3 \%$ & 13 & $15,6 \%$ \\
\hline Fossa seca & 32 & $38,5 \%$ & 7 & $8,4 \%$ \\
\hline
\end{tabular}

* $\mathrm{P}=$ parasitados; $\mathrm{F}$ = frequência; $\mathrm{NP}$ = não parasitados.

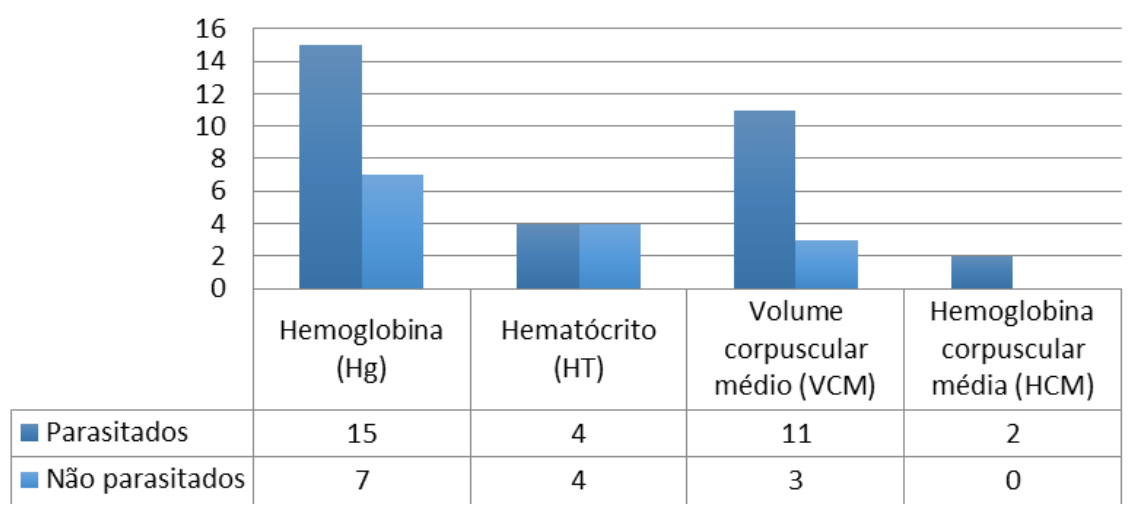

Gráfico 1. Îndices hematimétricos.

Observou-se uma maior prevalência de protozoários em relação aos helmintos, validando as resultas de Santos et al., (2017) onde é informado a prevalência de $80,8 \%$ de protozoários em proporção a $19,2 \%$ de helmintos e similar aos resultados obtidos em utilizadores do SUS no município de Ibiassucê por Matos e Cruz (2012) onde a taxa de protozoários estava acima de 93\%. Os resultados divergem de Araújo et al., (2020) que apresentou uma prevalência de 63,94\% para helmintos e 36,06\% para protozoários. Barçante et al., (2008) associa a redução da frequência desses agentes entre humanos ao uso de drogas anti-helmínticas. 
A ocorrência dos helmintos pode diversifica-se em sua periodicidade conforme as circunstâncias ambientais de cada localidade, afora as precárias condições de habitação, higiene pessoal e, sobretudo, a insuficiência de saneamento básico. Embora o A. lumbricoides e T. trichiura apresentarem vasta disseminação, tais patógenos são preeminentes em regiões de clima tropical e conjunturas sanitárias favoráveis à subsistência dos ovos, já que os mesmos perfazem seu ciclo de vida no solo. (Silva et al., 2019). Diversas pesquisas correferem as parasitoses intestinais à públicos rurais e urbanos, sexo, variedades sociais, condições a exposição de agentes infectantes e faixa etária.

Fundamentando-se no avantajado número de participantes das pertencentes faixas etárias que efetuaram os exames na época estudada, este estudo apresenta dados discordantes de Araújo (2017), que expõe que ocorre a queda da preponderância de enteroparasitos conforme o avançar da idade. Em relação ao sexo, houve uma maior incidência de parasitos intestinais no sexo feminino que no masculino, resultado semelhante ao apresentado por Cardoso et al., (2005). Tal presença dá-se devido às mulheres assumirem inúmeras tarefas domésticas que as expõe à contaminação por parasitos intestinais, principalmente quando habitam em localidades infectadas por dejetos, levando em conta que há uma maior procura aos serviços de saúde por parte das mesmas (Hurtado-Guerrero et al., 2005; Oliveira Filho et al., 2012).

Típicas em países subdesenvolvidos como Brasil, as parasitoses intestinais apresentam-se como uma adversidade comum em diversos países. Análises epidemiológicas de enteroparasitoses subsidiam informações referentes as práticas de higiene da população em estudo, dimensão do saneamento básico de tal região e nível de insalubridade da localidade. Conexa à contaminação orofecal, constituinte do mesmo condutor de transmissão de protozoários deletérios como a $G$. duodenalis, a assiduidade de protozoários comensais, embora não possua valor clínico apresenta relevância epidemiológica (Martins et al., 2014; Menezes et al., 2008).

Grande parte das pesquisas indica que a precariedade de hábitos higiênicos e de saneamento básico são fatores condicionantes à ocorrência da patologia, suplementarmente. Tais condições sofrem influência cultural e socioeconômica, já que alguns cuidados não são observados em populações mais carentes, ampliando as possibilidades de contaminação (Moraes et al., 2020; Jesus et al., 2013). Além disso, estes parasitos validam o déficit de saneamento básico, baixas condições socioeconômicas e de higiene, uma vez que indicam contaminação fecal-oral já que residem naturalmente no intestino de homens e animais (Bóia et al., 2006).

A existência de protozoários comensais no público foi marcante, salientando que, por mais que não acarretem doença parasitária, a detecção dessas formas em diagnósticos coproparasitológicos pode indicar o consumo de água contaminada por excretas humanas. Ademais, a forma de transmissão destes parasitos equivale ao utilizado pelos protozoários patogênicos com rota fecal-oral. O descarte e o tratamento adequado de material fecal reduzem significativamente os riscos de transmissão de protozoários e helmintos intestinais de transmissão fecal, entretanto, quando esse descarte é realizado em fossas rudimentares ou coleções hídricas reutilizadas sem tratamento adequado, aumenta-se consideravelmente o risco potencial de contaminação da população e de toda a comunidade (Magalhães et al., 2013).

Segundo Cavagnolli et al., (2015) o avanço socioeconômico de uma nação é reflexo da vulnerabilidade de sua população concernente a um saneamento básico insuficiente, baixa escolaridade e hábitos higiênicos deficitários. Em Água Branca, município do estado da Paraíba, as circunstâncias socioeconômicas são similares as da localidade do presente estudo e em pesquisa feita por Silva et al., (2014), observou-se positividade de $38,46 \%$ para parasitoses, com predominância de E. histolytica. Esse parasito, segundo Silva et al., (2014) evidencia-se por ser relevante fomentador de casos parasitários em escala global, assíduo em localidades que apresentam clima quente e úmido, coeso a meios insalubres.

Nunes et al., (2019) enfatiza que condições ambientais fragilizadas em decorrência da precariedade de moradias coletivas são aspectos iminentemente congruentes a propagação das verminoses, igualmente a carestia na qualidade da água, inapropriada destinação do lixo e a péssima acomodação dos excrementos. Os entrevistados utilizam água diretamente do rio para consumo próprio. A água é uma considerável ambiência de paraistoses de propagação hídrica e nas localidades que apresentam precárias condições de saneamento básico, os índices de parasitoses intestinais são expressivos.

Nessa perspectiva, faz-se necessária a edificação de redes de esgoto e estações destinadas ao tratamento da água, visando a prevenção das enfermidades de transporte hídrico. O solo e a água são notáveis meios de contaminação do ser humano, não obstante o próprio homem é responsável por contaminar o ambiente em 
que vive, uma vez que lança seus excrementos indevidamente. A aquisição de novos hospedeiros se dá através dos ovos, cistos e larvas que percorrem grandes espaços por meio aquático. Os casos de poliparasitismo tornam-se frequentes em populações expostas a um saneamento básico precário (Rocha et al., 2010).

Mesmo na eventualidade de relevantes e evidentes melhorias no que tange os hábitos higiênicosanitários e o saneamento básico, em especial nos grandes centros urbanos, a patologia ainda aflige muitos sujeitos, singularmente crianças, adolescentes, idosos e habitantes de áreas periféricas. Avaliar a prevalência dessas infecções é importante, pois ajuda a tomar medidas preventivas, de modo que haja controle de doenças associadas, reduzindo dessa forma os gastos com terapia especifica, ou seja, é necessária a análise visando fornecer informações para o delineamento de intervenções governamentais (Rocha et al., 2010; Chaves et al., 2020).

Pesquisadores têm indicado que a escolaridade, saneamento básico e fatores socioeconômicos são variáveis determinantes para aquisição de parasitoses. Sobre a constituição familiar, quando se tem famílias maiores, como grande parte das famílias da pesquisa, que apresentaram núcleo familiar de quatro pessoas ou mais, tem maior probabilidade de infecção parasitária. A disseminação, para algumas espécies, pode ocorrer pelo contato direto com indivíduos infectados. Por isso, muitas pessoas morando numa mesma residência, principalmente pequena, pode favorecer a contaminação (Chaves et al., 2020).

Aspectos diversificados favorecem a disseminação de parasitoses intestinais, essencialmente as conjunturas do saneamento básico. Outrossim, os malefícios causados por enteroparasitoses persistem no território nacional a despeito das melhorias que já ocorreram. Estudiosos consentem que educação em saúde associada ao sanemanto básico eficiente podem levar a redução da ocorrência de tal patologia (Bosqui et al., 2016).

\section{Considerações finais}

Com base nos resultados, foi observado um elevado numero de casos de parasitoses intestinais na população do bairro Jardim Tropical, município de Breves - PA, Amazônia Brasileira. Com isso, faz-se necessário a melhoria do saneamento básico, além do incentivo e educação em saúde para a população sobre os riscos e prevenção de parasitoses, promovendo assim, uma melhor qualidade de vida à comunidade.

\section{Referências}

Almeida, F. S., Silva, R. C., \& Medeiros, J. S. 2014. Ocorrência de helmintos e protozoários intestinais em idosos. Biofarm, 10(4), 78-82.

Araújo, J. R. O., Cavalcante, R. M. S., Carvalho Neto, A. P. M., Silva, K. W. L., Silva, S. R. G., Ferreira, J. R. S., ... Rocha, T. J. M. 2020. Frequency of intestinal parasites in patients attended at a university hospital in Maceió, state of Alagoas, Brazil. Bioscience Journal, 36(6), 2266-2274.

Araújo, V. A. D. 2007. Levantamento e aspectos epidemiológicos de helmintos em humanos no município de Seropédica, RJ. Revista Eletrônica Novo Enfoque, 5(5), 4-10.

Barçante, T. A., Cavalcanti, D. V., Silva, G. A. V., Lopess, P. B., Barros, R. F., Ribeiro, G. P., Neubert, L. F., \& Barçante, J. M. P. 2008. Enteroparasitos em crianças matriculadas em creches públicas do município de Vespasiano, Minas Gerais. Revista de Patologia Tropica, 37(1), 33-49.

Bóia, M. N., Costa, F. A. C., Sodré, F. C., Eyer-Silva, W. A., Lamas, C. C., Lyra, M. R., ... Carvalho, L. M. A. 2006. Mass treatment for intestinal helminthisis control in an Amazonian endemic area in Brazil. Revista do Instituto de Medicina Tropical de São Paulo, 48(4), 189-195.

Bosqui, L. R., Pereira, V. L., Custódio, L. A., Menezes, M. C. N. D., Murad, V. A., Almeida, R. S., ... Costa, I. N. 2016. Strongyloides stercoralis e outros parasitas intestinais na população humana da região norte do Paraná identificados utilizando diferentes métodos parasitológicos. Revista Brasileira de Análises Clínicas, 48(2), 153-9.

Cardoso, F. M., Morcillo, A. M., Zeferino, A. M. B., \& Antônio, M. Â. R. G. M. 2005. Enteroparasitoses em usuários da rede municipal de saúde de Campinas, São Paulo. Revista de Ciência Médica, 4(14), 337-344.

Castro, T. G., Silva-Nunes, M., Conde, W. L., Muniz, P. T., \& Cardoso, M. A. 2011. Anemia and iron defi ciency among schoolchildren in the Western Brazilian Amazon: prevalence and associated factors. Caderno de Saúde Pública, 1(27), 131-142. 
Cavagnolli, N. I., Camello, J. T., Tesser, S., Poeta, J., \& Rodrigues, A. D. 2015. Prevalência de enteroparasitoses e análise socioeconômica de escolares em Flores da Cunha-RS. Revista de Patologia Tropical, 44(3), 312-322.

Chaves, M. F., Dantas, S. H., Souza, S. A., Silva, A. B., Feitas, F. I. S., Cavalcante, U. M. B. \& Lima, C. M. B. L. 2020. Avaliação de variáveis sociodemográficas e da qualidade de vida de portadores de helmintiases intestinais em um hospital universitário. Revista Interdisciplinar de Estudos em Saúde da Uniarp, 9(1), 145-161.

Fraga, R. S. 2019. Desenvolvimento de anemia em pacientes com infecções parasitárias: revisão de literatura. Trabalho de conclusão de curso apresentado ao Curso de Bacharelado em Biomedicina. Governador Mangabeira, BA: Faculdade Maria Milza.

Hurtado-Guerrero, A. F., Alencar, F. H., \& Hurtado-Guerrero, J. C. 2005. Ocorrência de enteroparasitas na população geronte de Nova Olinda do Norte Amazonas, Brasil. Acta Amazonica, 35(4), 487-490.

Fundação Instituto Brasileiro de Geografia e Estatística [IBGE]. Breves. Disponível em: https://www.ibge.gov.br/cidades-e-estados/pa/breves.html. Acesso em: 12 fev. 2021.

Ignacio, C. F., Silva, M. E. C., Handam, N. B., Alencar, M. F. L., Sotero-Martins, A., Barata, M. M. L., \& Moraes Neto, A. H. A. 2017. Socioenvironmental conditions and intestinal parasitic infections in Brazilian urban slums: a cross-sectional study. Revista do Instituto de Medicina Tropical, 59(7), 1-10.

Jesus, J. S., Menezes, R. A. O., Andrade, R. F., Gomes, M. S. M., Barbosa, F. H. F., \& Faustino, S. M. M. 2013. Prevalência de enteroparasitoses em agricultores da feira do produtor rural do bairro do Buritizal, Macapá, Amapá, Brasil. Revista de Biologia e Ciências da Terra, 13 (2), 74-79.

Maia, C. V. A., Hassum, I. C., \& Valladares, G. S. 2015. Parasitoses intestinais em usuários do SUS em Limoeiro do Norte, Ceará,antes da expansão de sistema de esgotamento sanitário. Holos, 2(31), 98-109.

Magalhães, T. R., Coelho, M. D. G., Araújo, A. J. U. S., \& Coelho, F. A. S. 2013. Influência de fatores socioambientais na ocorrência de enteroparasitos e protozoários não patogênicos em área periférica do município de Cristina, MG - Brasil. Revista Biociências, 19(2), 18-26.

Martins, N. D., Cardoso, K. C. I., \& Couto, Á. A. R. D'A. 2014. Estudo da prevalência de enteroparasitoses no município de Ferreira Gomes/AP após a enchente em 2011. Biota Amazônia, 4(3), 15-24.

Menezes, A. L., Lima, V. M. P., Freitas, M. T. S., Rocha, M. O., Silva, E. F., \& Dolabella, S. S. 2008. Prevalence of intestinal parasites in children from public daycare centers in the city of Belo Horizonte, Minas Gerais, Brazil. Revista do Instituto de Medicina Tropical de São Paulo, 50(1), 57-59.

Moraes, L. J. R., Andrade, L. S., Farias, C. B. P., \& Pinto, L. C. 2020. Prevalência de anemia associada a parasitoses intestinais no território brasileiro: uma revisão sistemática. Revista Pan-Amazônica de Saúde, 10, e201900098.

Matos, M. A., \& Cruz, Z. V. Prevalência das parasitoses intestinais no município de Ibiassucê - Bahia. 2012. Revista Educação, Meio Ambiente e Saude, 1(5), 64-71.

Naoum, P. C., \& Naoum, F. A. 2011. Diagnóstico diferencial das anemias microcíticas e hipocrômicas. Revista Brasileira de Análises Clínica, 43(3), 160-162.

Nunes, M. O., \& Rocha, T. J. M. 2019. Fatores condicionantes para a ocorrência de parasitoses entéricas de adolescentes. Journal Of Health \& Biological Sciences, 7(3), 265-270.

Oliveira Filho, A. A., Abrantes, H. F. L., Fernandes, H. M. B., Viana, W. P., Pinto, M. S. A., Cavalcanti, A. L., \& Freitas, F. I. S. 2012. Perfil enteroparasitológico dos habitantes de uma cidade do Nordeste do Brasil. Revista Brasileira de Clínica Médica, 3(10), 179-182.

Rocha, T. J. M., Braz, J. C., \& Calheiros, C. M. L. 2011. Parasitismo intestinal em uma comunidade carente do município de Barra de Santo Antônio, Estado de Alagoas. Revista Eletrônica de Farmácia, 7(3), 28-33.

Santos Júnior, J. G. A., Cruz Neto, P. A., Cristo, J. S., \& Vandesmet, V. C. S. 2016. Anemia associada às parasitoses intestinais de pacientes atendidos em um laboratório de análises clínicas no município de Juazeiro do Norte - Ce. Revista Interfaces: Saúde, humanas e tecnologia, Juazeiro do Norte, 3(9), 06-03.

Santos, P. H. S., Barros, R. C. S., Gomes, K. V. G., Nery, A. A., \& Casotti, C. A. 2017. Prevalence of intestinal parasitosis and associated factors among the elderly. Revista Brasileira de Geriatria e Gerontologia, 20(2), 244-253.

Silva, A. M. B., Bouth, R. C., Costa, K. S., Carvalho, D. C., Hirai, K. E., Prado, R. R., Araújo, S. G., Pereira, A. C. L., Ribeiro, K. T. S. 2014. Ocorrência de enteroparasitoses em comunidades ribeirinhas do Município de Igarapé Miri, Estado do Pará, Brasil. Revista Pan-Amazônica de Saúde, 5(4), 45-51.

Silva, R. S. B., Malheiros, A. F., Santos, D. P., Shaw, J. J., Araújo, M. S. M., Moraes, M. F. A., \& Campos, W. N. L.. 2019. Estudo de parasitoses intestinais em moradores de corumbá, Mato Grosso do Sul, Brasil. Revista Ibero Americana de Ciências Ambientais, 10(2), 109- 128. 
Sucasas, H. T. F., Faria, F. C., Costa, E. G. D., \& Silva, J. S. 2017. Avaliação parasitológica e análise de hemograma de crianças do projeto Novo Horizonte no município de Manhaçu, MG. In III SEMINÁRIO CIENTÍFICO DA FACIG. Manhuaçu, MG.

Teles, M. F. P. 2018. Anemia ferropiva associada à infecção por Ancilostomídeo. Trabalho de conclusão de curso apresentado ao Curso de Bacharelado em Biomedicina. Centro Universitário São Lucas, Porto Velho, RO: Centro Universitário São Lucas.

\section{Minicurrículo}

Emilly Gabriele Prata de Abreu. Acadêmica de Enfermagem e Bolsista de Iniciação Cientifica PROBIC da Universidade Federal do Amapá, Macapá - Amapá. Brasil.

Max Amaral Balieiro. Acadêmico de Enfermagem da Universidade Federal do Amapá, Macapá - Amapá. Brasil.

Klingerry da Silva Pennafort. Universidade Federal do Amapá (Unifap). Docente do curso de Enfermagem e Mestre em Ciências da Saúde. Macapá - Amapá. Brasil.

Camila Rodrigues Barbosa Nemer. Docente de enfermagem da Universidade Federal do Amapá (Unifap). Docente do curso de Enfermagem e Mestre em Enfermagem. Macapá - Amapá. Brasil.

Edmundo de Souza Moura Filho. Docente de enfermagem da Universidade Federal do Amapá (Unifap). Especialista em Saúde Pública. Macapá - Amapá. Brasil.

Rubens Alex de Oliveira Menezes. Graduação em Enfermagem (Bacharelado e Licenciatura) pela Universidade Federal do Amapá (2009). Pós-Graduação em Microbiologia Nível de Especialização pela Universidade de Fortaleza, Pós-Graduação em Docência do Ensino Superior pela Faculdade de Tecnologia do Amapá - META, Pós-graduação em Gestão em saúde pública pela Faculdade de Macapá - FAMA, PósGraduação Nível de Mestrado em Ciências da Saúde Área de Concentração: Epidemiologia e Saúde Pública pela Universidade Federal do Amapá (2013). Pós-graduação Nível de Doutorado em Biologia de Agentes Infecciosos e Parasitários (PPG BAIP) pela Universidade Federal do Pará. Atualmente é docente do curso de enfermagem da Universidade Federal do Amapá (UNIFAP), Adjunto I, atuando na linha de pesquisa direcionada as doenças emergentes, reemergentes e negligenciadas, desenvolvendo estudos com enfoque especial em: Saúde pública e epidemiologia e controle de doenças transmissíveis, não transmissíveis, infecciosas e parasitárias de interesse médico-sanitário.

Como citar: Abreu, E.G.P., Balieiro, M.A, Pennafort, K.S., Nemer, C.R.B., Moura Filho, E.D., \& Menezes, R.A.O. 2021. Índices hematimétricos associado as parasitoses intestinais no Município de Breves, Ilha do Marajó, Pará. Pubsaúde, 7, a210. DOI: https://dx.doi.org/10.31533/pubsaude7.a210

Recebido: 8 jun. 2021.

Revisado e aceito: 24 jun. 2021.

Conflito de interesse: os autores declaram, em relação aos produtos e companhias descritos nesse artigo, não ter interesses associativos, comerciais, de propriedade ou financeiros que representem conflito de interesse.

Licenciamento: Este artigo é publicado na modalidade Acesso Aberto sob a licença Creative Commons Atribuição 4.0 (CC-BY 4.0). 